\title{
History of English Translations and its Influence on Nepali Literature
}

\begin{abstract}
:
Translation has been one of the instrumental factors in enriching the Nepali Polysystem from its inception. The translation of the Adhyatma Ramayana by Bhanu Bhakta Acharya helped to establish the literary culture in Nepali language. Since then Nepali literary culture has been borrowing various other genres, themes and aspects from English language. Regardless of these, translation has been looked down as a secondary process; hence the paper would explore the variegated translations from English into Nepali which has enriched the target culture. Therefore, the paper would provide the lists of translations which have been done in Nepali from English. The range of translation is limited to few genres: Poetry, Novels, Short Stories and Drama.
\end{abstract}

Sudesh Manger

Key Words: Translation, Nepali Literature and History of Translation

The growing interest in documenting the history of translation shown by several scholars in the recent years motivated me to document the history of translation' in Nepali literature. By documenting the history of translation, the ground is prepared for retrospective and reflective engagement with the development of discourses in Nepali translation. A vivid picture of the past can act as a foundation for the present; producing a sort of "touchstone method" to analyze the translations of the present generation. Therefore, the aim of this chapter is to reflect on the views and the achievements of the translators of the past and their influences on Nepali writers. The chapter contains my personal translation of two 
Nepali texts Anuvad Bimrsh (2010) by Raj kumari Dhal and Sahitya ko Itihas: Sidhanta ra Sandarva (2002) by Dayaram Shresta. The Nepali texts provide the entire list of English translations into Nepali as per decades; however, the present research has categorized the list as per genre to deal with the influences that English translation brought in a particular genre.

To consider the overall influence of the English translations on Nepali literature, different genres are discussed separately. The Nepali poetry being one of the earliest forms of literature has been influenced by various western poets or schools of poetry like Romanticism, Heroic poetry, Modernism etc. Consequently, Nepali poets embellished their writings better than earlier. The revolutionary ideas of Romanticism against the Industrialization were used to write against the ruling government of Nepal. Lekhnath Paudel's Pinjara ko Suga (A parrot in a Cage, 1962) is notable among such attempts. Lakshmi Prasad Devkota (1909-59) and Lekhnath Paudel show the romantic elements in their poems. Muna Madan (1943) of Devkot depicts the hardship of life in the industrial world through Madan's struggle in Lassa (Tibet). Ishwar Balav's prose poem Avaj Kaham Cha? (Where Is the Voice?)(1962), depicts the hippocratic contemporary society similar to the modernists like T.S Elliot. Drama and play, the neglected genres in Nepali, also saw the pinnacle of success thorough the influence of Brecht, Henrik Ibsen, Anton Chekov, William Shakespeare etc. Balkrishna Sama wrote several dramas based on the model of Shakespearean plays like Muto Ko Byatha (Heart Ache, 1965). Novels and short stories, the two genres which are the adaptations from the western model of writing completely changed the face of Nepali literature and they started to occupy the central position in Nepali literature. 
Apart from the adaptations, the borrowed themes like Existentialism, Modernism, Feminism, etc, also started to occupy the central position in the Nepali literature. This is evident in the writings of the famous Nepali novelists like Parijat (e.g., Sirsha ko Phul (Blue Memosa, 1965) and Boni (1991)) and Bishweshwar Prasad Koirala (e.g., Sipahi (A Soldier, 1935)). Highlighting the influential aspect of translation Dayaram Shresta in Sahitya ko Itihas (History Of Nepali Literature) says:

"Bivinna Bhasaharu bata Nepali ma Anuvad gareya ka kritiharule ekatira itahas nirman ma sayog purayo bhane arko tiro moulik lekhanka nimti pratibha harulai abhiprerit are..... yas ko sathye ti anuvadakharule samaj ko sanskritik ra lokpriyata ko pani paripurit garediya. Yashari adhunikal purva ko nepali anuvadak sahitya sandesh mulak raheko paincha. (Shresta, 2008, 117)"

"The translation from different languages helped to develop the national literature; at the same time it encouraged the writers with new ideas. To draw a conclusion about the translational activity till 1937, we can say that the scholars from both Nepal and India studied different languages and literatures, while reading whatever classics they found in other literature, they translated them into Nepali. By doing so they filled the literary vacuum of Nepali literature. The thought of bringing the classics from other literatures to enrich their own literature is one of the best aspects of translation in Nepali literature. (Translation is mine)"

The act of historicizing the translational project is not a new phenomenon, it has been done by several scholars in the world like, Paul Horguelin at the Universite de Montreal in the early 1970's, and Jean Delisle and Louis Kelly have been teaching history since the mid-1970s at the University of Ottawa, School of Translators and Interpreters. Samuel Johnson's The Idler (1759) traces the history of translation from Ancient Greece to the seventeenth- 
century England, to illustrate the triumph of non-literal translation. However, the role of translation in Nepali literature from English as per my investigation has not been fully explored. And most of the scholars who worked in this area have focused on either Nepali literature of Nepal or Nepali literature of India. The present chapter, therefore, will try to present conglomerate efforts of various scholars to develop the unified Nepali literature. The process of writing the history of translation has been appreciated by various translation scholars, and Antonio Berman believes "the construction of a history of translation is the most pressing task of a truly modern theory of translation" (Berman, 1984, 12) and he states "It's time to give the history of translation the place it deserves." (Berman, 1984, 12)

Berman talks about the attention that translation deserves which has not been given in most of the academia. He is aware of the undeniable contribution of translation as a building block of a literature in a given culture. It was one of the major sources used to enrich the literature during the formative period, as no culture or literature could be self-sufficient in itself; it had to rely on the material from the foreign sources. In terms of Nepali literature, the traces of translation in the ancient time are very few and can be found in slokas, tamrapatra, stones and pillars engraves. During the early $19^{\text {th }}$ century, scholars like Motiram Bhatta started to document the evidences found in written form. As such, the written evidences could be found mostly after the publication of an edited version of Bhanu Bhakta's translation of The Ramayana from Sanskrit in 1816. This shows that the written form in Nepali literature is not a very old phenomenon; it was mostly dominated by the Dantya Khata or Oral literature. 


\section{The History of Translational Activity from English into Nepali}

The influence of colonialism has changed the face of the earth not only geographically and economically but also socially, religiously, politically and linguistically. Though politically colonialism did not take place in Nepal, nevertheless it had a colonial influence on its language, literature and religion. The introduction of various English medium schools and colleges helped produce scholars, who started to write in English. The emphasis in English education led to the proliferation of English speaking people among the Nepali community which helped embellish the Nepali language by borrowing from English.

While the language and literature brought a shift in academics, the religion changed the socio-cultural aspect of Nepali community. Several Hindu communities proselytized themselves into Christianity and started spreading Christianity through the translation of the Bible into the regional language. Therefore, the influence of English language and education can be seen through the proliferation of translations from English into Nepali.

The advent of modern literature in Nepal began in the 1920s and 30s with the work of Balkrishna Sama, who wrote lyrical poetry, plays based on Sanskrit and English models, and also some short stories. Sama and his contemporary, the poet Lakshmiprasad Devkota, discarded the earlier Sanskrit-dominated literary tradition and adopted some literary forms of the West, notably prose poetry, tragic drama, and the short story. In their poetry, these writers dealt with such themes as love and patriotism as well as the problems of injustice, tyranny and poverty faced by Nepal in the $20^{\text {th }}$ century. Modern Nepali drama, of which Sama was the chief practitioner, was influenced in its depiction of contemporary social problems by 
Western playwrights, notably Henrik Ibsen. In the hands of such writers as Bisweswar Prasad Koirala and Bhavani Bhiksu, the Nepali short story also centered on social problems of modern-day Nepal and the need for reformation. The selected texts for case study are not dealt in detail here as the purpose is only to highlight their influence on the major writings in Nepali.

\section{Translation of Poetry}

Poetry is one of the oldest and important genres of Nepali literature from time immemorial; it is looked upon as a national treasure by most of the scholars. The focus on the influence of translations on Nepali poetry is not an attempt to undermine the potentialities of the Nepali poets, but to look at how they have incorporated the novel thoughts and ideas that they were introduced through translations in their regular pattern of writings. When someone is trying to deal with poetry, the influence of romanticism, Shakespearean sonnets, Modernist poets cannot be ignored; similarly these schools have also influenced the way of writing in Nepali poetries. The influence of Sanskrit, Hindi and Bengali is apparent in the poets like Bhanu Bhakta, Moti Ram Bhatta, Suvananda Das, Gumani Panta, Yadunath Pokhrel, Pahalmansingh Swanr, Sikharnath Subedi and Rajiblochan Joshi. These were the poets who were enmeshed in writing religious and war poetries. The trend of Bhakti Dhara and Veerdhara continued till the early $20^{\text {th }}$ Century.

The herculean task of moving out of the clutches of the Bhakti and Veerdhara pattern was initiated by the poets like Lakshmi Prasad Devkota, Lekhnath Paudel, Bhim Nidhi Tiwari, Bairaqi Kainla, Balkrishna Sama, Ishwar Balav and Gopal Prasad Rimal. The effect of English Modernist writings, especially prose poems can be seen in Gopal Prasad Rimal's collection of poems called 
Gumne Mechmathi Andho Manche (A Blind Man on a Revolving Chair: 1962) which is one of the most important contributions in Nepali literature. Bairaqi Kaila and Ishwar Balav through Tesro Ayam or the third dimension movement wrote on a journey into their own psyches, about what they felt was lacking in the usage of the Nepali language. A third dimension is where one writes without inhibitions, spontaneously portraying one's innermost feelings through a psychological, cultural, and socio-archetypal process. This movement changed the conventional way in which Nepalese literature was seen and gave the readers new insights into the minds of the poets and writers. The poems of Bhim Nidhi Tiwari which deal with the social issues like smoking, drinking, and gambling were highlighted for the first time through his poems like Dagbatti (Funeral Lamp: 1957).

Though most of the poets played a major role in shaping the Nepali literature, the focus of the present research is on the poetry of Bairaqi Kaila and the influence of Modernism and Emily Dickinson in his prose poems. Bairaqi Kaila is one of the most influential writers of the time and his poems read like the poetries of T.S Elliot's The Waste Land (1922) which is written in the form of prose poetry. Both the poets belong to the modernist group. Elliot deals with the aftermath of the World War I and the catastrophe that ravaged the human lives.

Kaila deals with the contemporary hopeless society. Like Eliot he feels that a drunken man is better than a sane man, at least he does not harm anyone other than himself. Mateko Mancheko Bhashan: Madhyaratpachiko Sadaksita (A Drunk Man's Speech to the Street after Midnight 1979) shows the hopelessness of the conscious people who act like deaf and dumb to the present deteriorating society which is in the hands of a few political leaders. 
The lines below show his frustration with the contemporary society which is similar to what Eliot expressed in his poems.
All my steps are earthquakes today,
Volcanoes erupt in each sensation;
How have I lived to such an age
in these cramped and crumbling houses,
Too small for a single stride?
I am saddened:
Even now they sleep, self-defeated men,
Tangled together like worms
In the pestilent houses of the earth,
And do they sleep so late? (Hutt, 1991, 104)
What are the roots that clutch, what branches grow
Out of this stony rubbish? Son of man,
You cannot say, or guess, for you know only
A heap of broken images, where the sun beats,
And the dead tree gives no shelter, the cricket no relief,
And the dry stone no sound of water. (Eliot, 1934, 29)

The influence of Eliot is evident in the above lines; the theme and the style are quite similar. While dealing with the influence of Modernism on Nepali literature, one cannot ignore Emily Dickinson. The theme of death and immorality of Emily Dickinson is well incorporated by Baraqui Kailan in his poem, Sapna ko Las (The Corpse of a Dream 1965) which reverberates the poems of Dickinson. The lines below clearly show the similarity between the two poems:

My love,

A dream should last the whole night long.

My breast is where I sleep at nights,

Covered by vest and blouse,

Like an old man's cave inside a village

Where only the jackal and the fox

Call out their evil omens. 
Ruthlessly it is beaten

By bundles of office files

Which must be revealed to others,

By crises of convention,

The absence of choice. (Hutt, 1991, 102)

We slowly drove, he knew no haste,

And I had put away

My labour, and my leisure too,

For his civility. (Selected poems of Emily

Dickenson)

The influence of Modernism on Nepali literature gave a new dimension to the poets of Tesro Ayam who experimented with various themes and styles. These changes and influences were not only revolutionary but also most contributive towards the development of Nepali literature. The socio-cultural, historical and political situations do have their own influences in bringing shifts in the trend of poetry writing, but the translation of English poetry which complies with their need also played a major role in shaping their writings. Therefore to show the influence a list of English poetries which have been translated into Nepali are given below. Though the number of poetries translated from English is small, their influence has been vast. The classical poetry of the world literature translated from English to Nepali also played a major role in shaping the Nepali poetry. Given below is the list of poems translated from English into Nepali: 


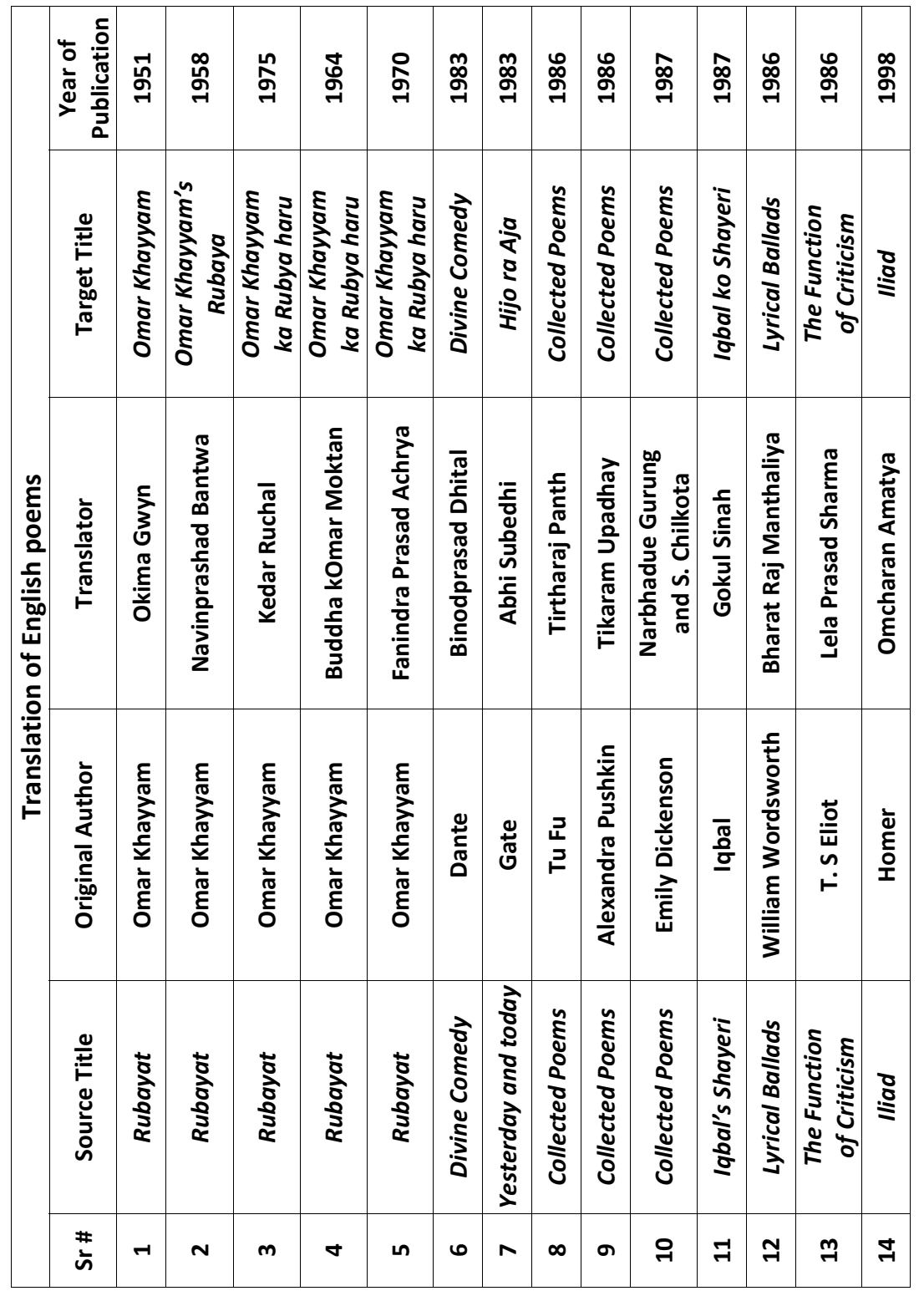


Though the number of poems translated from English is very few in number but the roles these translations played are immense. The Rubayat of Omar Khayyam has been translated by five different translators at different time frame. The Collected poems of Emily Dickinson is still considered as one of the best translations in Nepali literature as a recognition, the University of Tribhuan (Katmandu) included in their university curriculum. The purpose of translating classical texts like Dante's Divine Comedy and Homer's Iliad was to collect the canonical texts from the European literature.

\section{Translation of Novels and Short Stories}

Nepali literature was dominated by poetry and dramas, until the advent of the two new genres, novel and short story, and these two have changed the literary scenario completely, as they started occupying the central position pushing the poetry and dramas to the periphery. Apart from the new genres, the writers also borrowed the themes like, Modernism, Existentialism, and Feminism. The modernist thought of inner consciousness influenced the writings of Indra Bhadur Rai who published a philosophical text, Leela Lekhan ${ }^{2}$ which was considered to be one of the first philosophical texts in Nepali literature during Tesro Ayam movement in Darjeeling. Modernists' thought brought the philosophical writings into Nepali literature. Influence of translation can also be seen through the proliferation of feminist writers like Parijat, Bindya Subba, Manjushree Thapa and Dev Kumari Thapa.

The influence of Realism through translation of some of the Russian writers can be seen in the writings of Bishweswar Prasad Koirala. Several of his stories bear a striking resemblance to those of Chekhov, who draws attention to the very simple things of life and 
the joys and sorrows in the lives of common people. For instance, his Doshi Chashma (Defective Glasses: 1949), in some respects, is closer to the story line of Chekhov's Death of a Clerk (1883). Bishweswar Prasad was influenced by the realist Indian writers and also by the Russian authors such as Turgenev, Dostoyevsky, Tolstoy, and the French realists Maupassant and Anatole France. But the strongest influence on him was by the twentieth-century psychologists, especially Freud. Bishweswar Prasad wrote psychological stories relating to the problems of women, sex and the sadness of life. This style of story writing was new in Nepali literature, and for this reason he became very popular. Psychological aspects related to social problems such as marriages between childbrides and middle-aged grooms, untouchability, caste and class conflicts, and exploitation of the poor and the have-nots by the feudal class are brilliantly depicted by Koirala, along with a touch of satire.

The literature which has been highly dominated by male writers saw the emergence of women writers who depicted the various aspects of their situation in Nepali society. Komara Thapa deals with the social injustices through children literature and her most influential book Vara Parabata (Brave Mountain: 1976) shows the harsh reality of Darjeeling. Manjushree through her novels in English caters to the larger audience and gives the global exposure to Nepali literature. Parijat is one of the most influential women writers who changed the entire shape of Nepali writings by making women as her central character in her novels like Sirsha ko Phul (Blue Memosa) and Boni. The influence of the existentialist writers is very much evident in her novels.

Blue Memosa is based on the story of a young girl, Bari who is suffering from cancer but does not care much about death and 
continues smoking. The existentialist character of Parijat has been highly influenced by Fyodor Dostoyevesky and Franz Kafka. Free will and individual freedom are important to Bari which is evident in the lines below:

If a flower buds for itself and opens for itself and, as if accepting some complication, falls whether it fights the black bee or not, then why should it fall suffering the sting of the black bee? It falls only for itself. It falls by its own will. (Parijat, 2010, 14)

In Blue Mimosa, Bari doesn't believe in such predetermined essence. She even challenges rationality that defines God, good or bad, and the condition of being. For her, religion and the idea of god are arbitrary. Regarding God's existence she says "Don't say 'god', say 'the idea of god'. It's only a concept" and "washing away one's sins before the idea of god is completely meaningless" (Parijat, 2010, 37). A central proposition of existentialism is that existence precedes essence, which means that the actual life of the individual is what constitutes what could be called his or her 'essence' instead of there being a predetermined essence that defines what it is to be a human. The new way of dealing with the characters has given a new dimension, which has been an influential factor for Bindya Subba who modeled her novel Athah (Unfathomable: 2010) with various characters engrossed in their own lives. So, the translated novels have been able to influence the contemporary writings of the novels. The list of novels and short stories translated from English is given below: 


\begin{tabular}{|c|c|c|c|c|c|c|c|c|c|c|c|c|c|c|}
\hline & \multicolumn{2}{|c|}{ 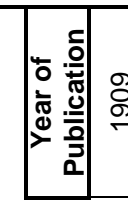 } & : & 구 & $\begin{array}{l}\stackrel{9}{9} \\
\stackrel{-}{\rightarrow}\end{array}$ & 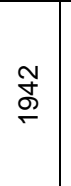 & 今ั & $\underset{\sim}{\stackrel{\Im}{9}}$ & 导 & $\stackrel{\infty}{\stackrel{\leftrightarrow}{\hookrightarrow}}$ & ఫ্ণ & $\underset{ণ}{\stackrel{一}{~}}$ & 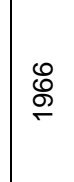 & 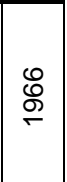 \\
\hline \multirow{2}{*}{ 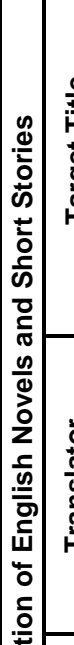 } & 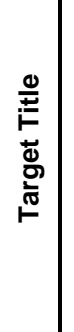 & 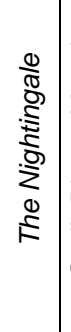 & 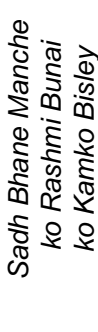 & 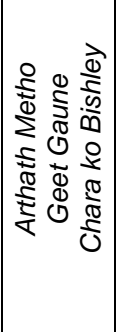 & $\frac{\Phi}{\omega}$ & 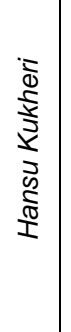 & $\begin{array}{l}8 \\
8 \\
0 \\
0 \\
0 \\
\frac{0}{4} \\
\mathbb{8} \\
0\end{array}$ & $\begin{array}{c}\frac{\pi}{8} \\
\mathbb{Q} \\
\mathbb{Q} \\
\frac{0}{U} \\
\frac{U}{U}\end{array}$ & 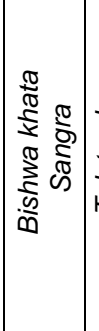 & 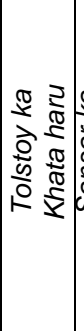 & 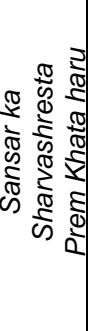 & 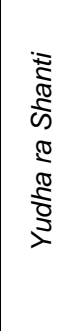 & 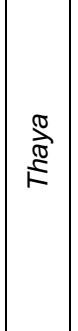 & $\begin{array}{l}\bar{b} \\
\overline{8} \\
\frac{1}{0} \\
0 \\
\frac{1}{\pi} \\
\frac{\pi}{0} \\
\bar{d} \\
0\end{array}$ \\
\hline & 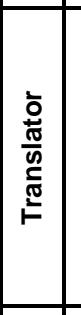 & 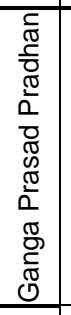 & 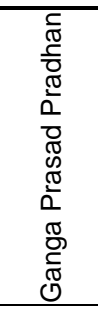 & 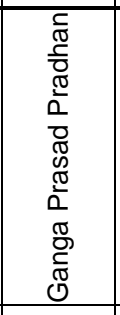 & 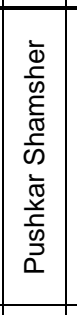 & 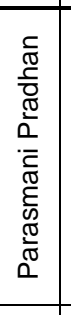 & 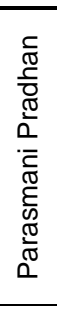 & 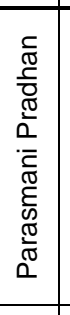 & 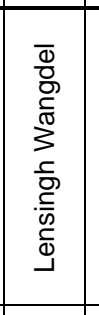 & $\begin{array}{l}\frac{0}{\pi} \\
\frac{\pi}{0} \\
0 \\
0 \\
0 \\
\frac{\pi}{0} \\
\underline{0}\end{array}$ & 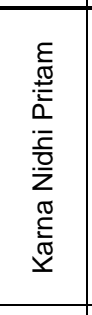 & 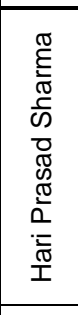 & 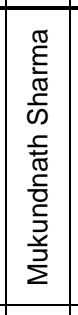 & 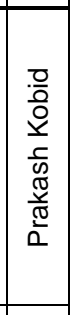 \\
\hline 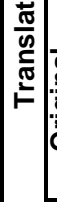 & 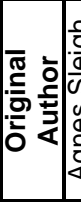 & 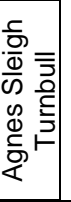 & $\begin{array}{l}8 \\
0 \\
0 \\
0 \\
0\end{array}$ & $\begin{array}{l}\text { O̊ } \\
0 \\
0 \\
0\end{array}$ & 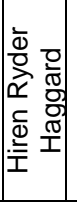 & & & & & 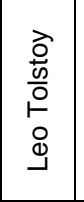 & ' & $\begin{array}{l}\frac{\overrightarrow{0}}{0} \\
\frac{0}{0} \\
\stackrel{0}{\circ} \\
0 \\
\Phi \\
\end{array}$ & 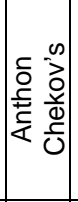 & 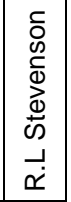 \\
\hline & 岕 & 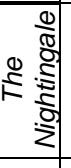 & 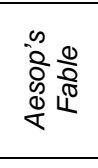 & 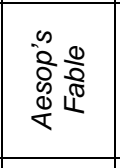 & $\frac{d}{\omega}$ & 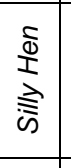 & 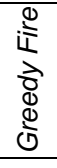 & 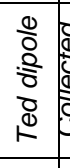 & 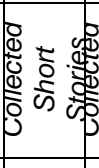 & $t$ & 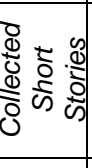 & 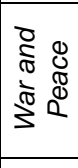 & 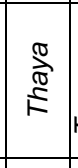 & 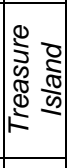 \\
\hline & $\begin{array}{l}\# \\
\dot{\omega}\end{array}$ & $\neg$ & $N$ & $m$ & $\nabla$ & เ & 0 & $r$ & $\infty$ & $\sigma$ & 우 & $\exists$ & ㄱ & $\stackrel{m}{-}$ \\
\hline
\end{tabular}


History of English Translations and its Influence on Nepali Literature

\begin{tabular}{|c|c|c|c|c|c|c|c|c|c|c|c|c|c|}
\hline & 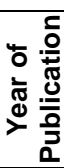 & $\begin{array}{l}\stackrel{8}{\circ} \\
\stackrel{\leftrightarrow}{-}\end{array}$ & 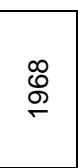 & 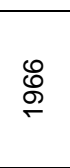 & $\begin{array}{l}\stackrel{8}{\circ} \\
\stackrel{-}{-}\end{array}$ & ત્ન & 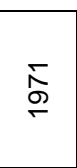 & స్త్ & స్ & $\underset{ }{\tilde{N}}$ & $\underset{\sigma}{\tilde{\sigma}}$ & స్ & 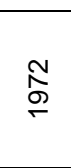 \\
\hline & 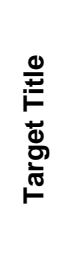 & 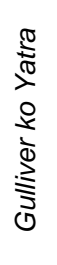 & 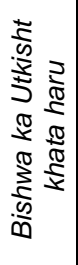 & 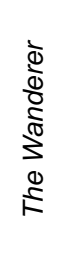 & 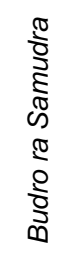 & 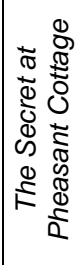 & 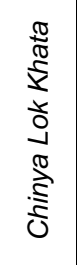 & 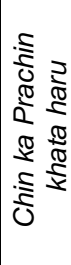 & $\begin{array}{l}\mathbb{\Xi} \\
\mathbb{\infty}\end{array}$ & 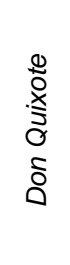 & 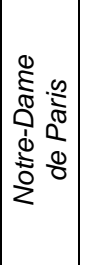 & $\begin{array}{l}\frac{1}{3} \\
\frac{5}{2} \\
0 \\
\frac{0}{2} \\
\frac{\vec{d}}{\omega} \\
\frac{j}{\omega}\end{array}$ & 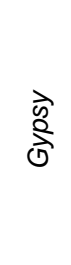 \\
\hline 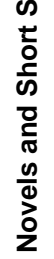 & 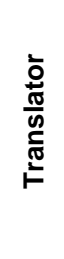 & 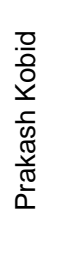 & 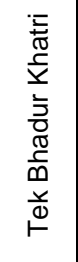 & 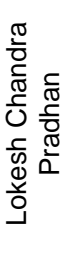 & 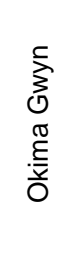 & 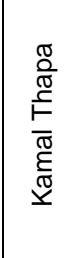 & 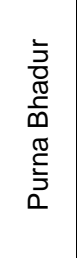 & 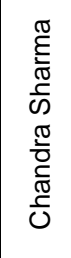 & 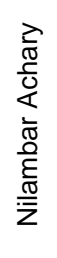 & 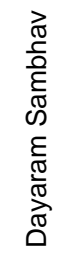 & 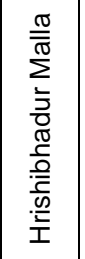 & 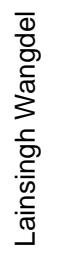 & 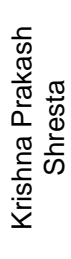 \\
\hline ; & 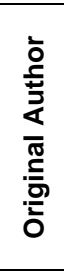 & 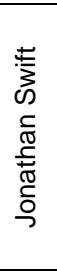 & ' & 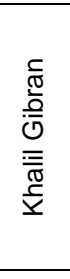 & 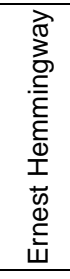 & 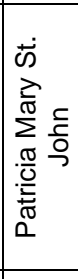 & ' & & 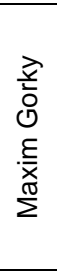 & 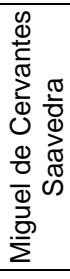 & 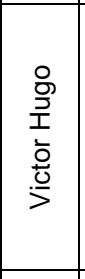 & 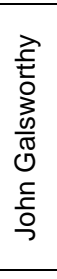 & 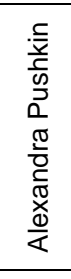 \\
\hline & 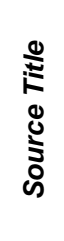 & 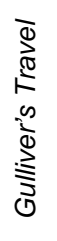 & 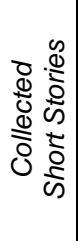 & 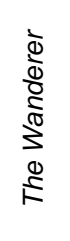 & 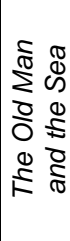 & 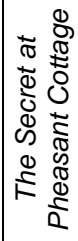 & 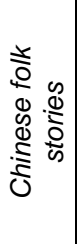 & 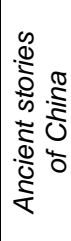 & 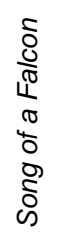 & 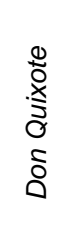 & 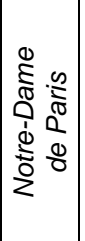 & 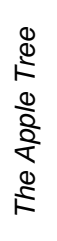 & ڤे \\
\hline & $\bar{n}$ & -1 & صـ & $\stackrel{0}{-}$ & ન્ & $\stackrel{\infty}{-1}$ & ને & $\stackrel{\sim}{ }$ & $\vec{N}$ & $\approx$ & $\stackrel{m}{N}$ & $\stackrel{\sim}{\sim}$ & \\
\hline
\end{tabular}




\begin{tabular}{|c|c|c|c|c|c|c|c|c|c|c|c|c|c|}
\hline & 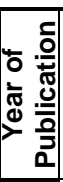 & $\stackrel{\text { م}}{\stackrel{\leftrightarrow}{G}}$ & \begin{tabular}{l}
0 \\
$\stackrel{2}{-}$ \\
\multirow{-}{*}{}
\end{tabular} & $\begin{array}{l}0 \\
\stackrel{2}{9} \\
-7\end{array}$ & $\left|\begin{array}{l}0 \\
2 \\
0 \\
-1\end{array}\right|$ & $\begin{array}{l}\stackrel{2}{2} \\
\stackrel{-}{9}\end{array}$ & $\mid \begin{array}{c}0 \\
\infty \\
\stackrel{్}{\rightarrow}\end{array}$ & $\begin{array}{l}\mathscr{0} \\
\stackrel{\circ}{\rightarrow} \\
\rightarrow\end{array}$ & 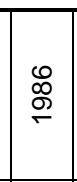 & $\begin{array}{l}\text { o } \\
\text { g }\end{array}$ & $\begin{array}{l}\stackrel{8}{\infty} \\
\stackrel{\rightarrow}{\rightarrow-}\end{array}$ & $\begin{array}{l}\stackrel{2}{\circ} \\
\stackrel{-}{\rightarrow}\end{array}$ & 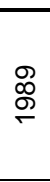 \\
\hline & 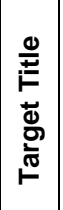 & 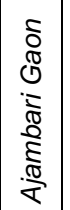 & 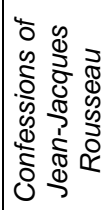 & है & $\left|\begin{array}{l}\tilde{z} \\
\hat{\xi} \\
\hat{\psi}\end{array}\right|$ & 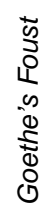 & 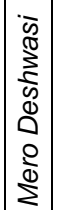 & 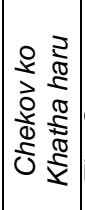 & 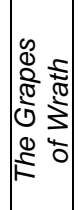 & 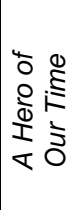 & 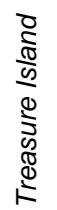 & 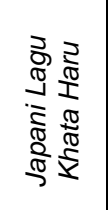 & 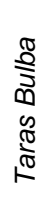 \\
\hline 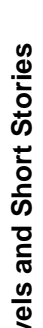 & 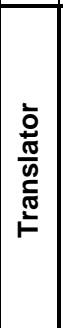 & 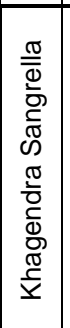 & 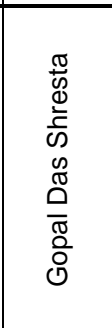 & 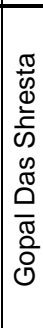 & 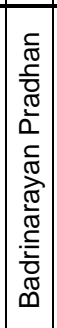 & 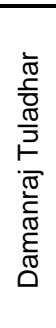 & 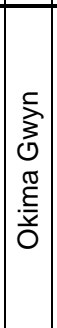 & 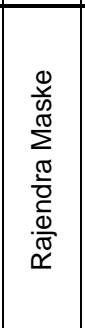 & 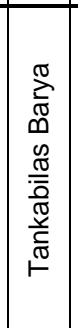 & 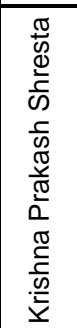 & 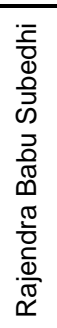 & 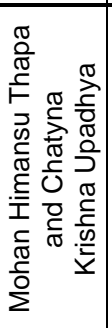 & 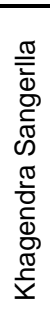 \\
\hline 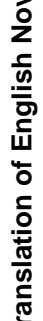 & 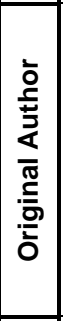 & 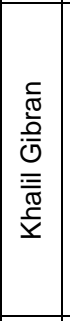 & 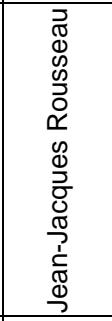 & 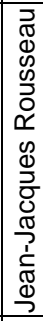 & 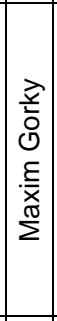 & $\begin{array}{l}\stackrel{0}{ \pm} \\
\stackrel{ \pm}{0} \\
0 \\
0\end{array}$ & 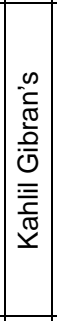 & $\begin{array}{l}\frac{\partial}{0} \\
\frac{0}{d} \\
\text { U } \\
\frac{5}{0} \\
\frac{0}{4}\end{array}$ & 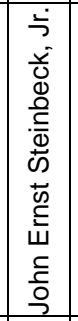 & 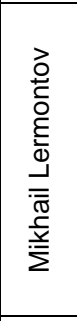 & 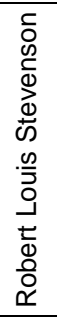 & & 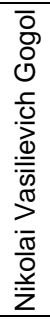 \\
\hline & 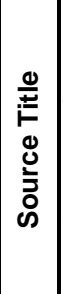 & 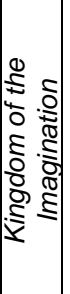 & 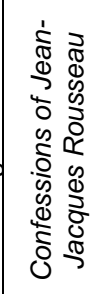 & 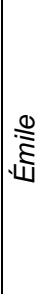 & $\mid \begin{array}{l}\bar{\Phi} \\
\bar{\Xi} \\
\sum\end{array}$ & $\begin{array}{l}\overline{\widetilde{Y}} \\
\stackrel{0}{L}\end{array}$ & 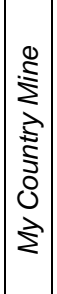 & 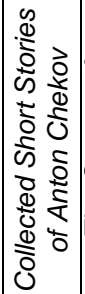 & 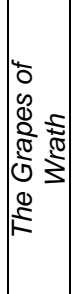 & 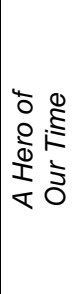 & 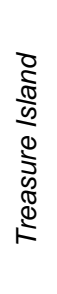 & 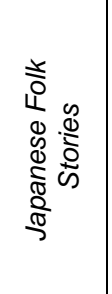 & 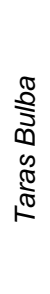 \\
\hline & $\begin{array}{l}\# \\
\dot{\omega}\end{array}$ & $\stackrel{\sim}{\sim}$ & $\grave{N}$ & $\stackrel{\infty}{\sim}$ & $\mathbb{N}$ & ిల & $\vec{m}$ & $\tilde{m}$ & ల్ల & ले & $\stackrel{m}{m}$ & $\stackrel{m}{\infty}$ & $\hat{m}$ \\
\hline
\end{tabular}




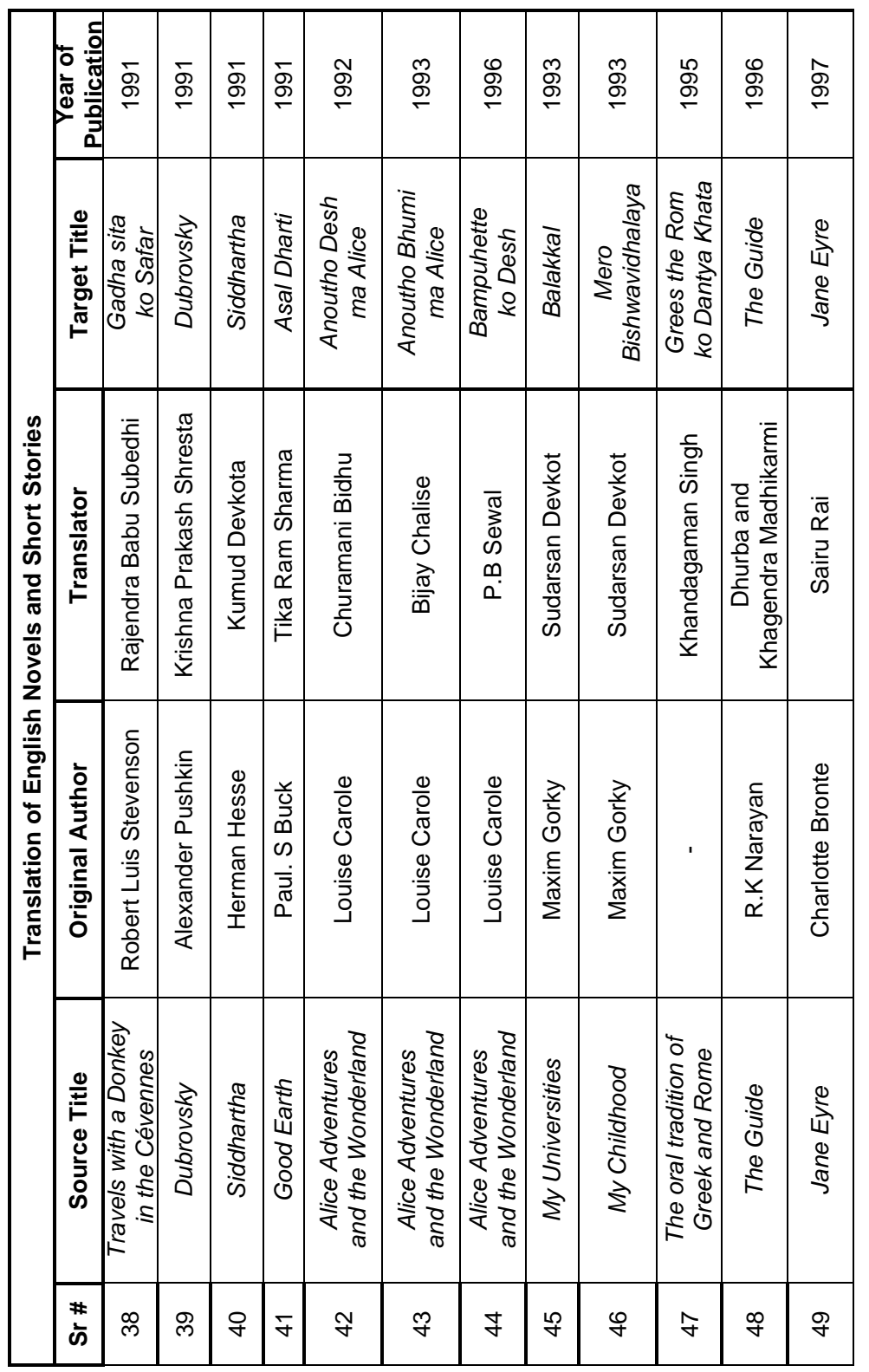




\begin{tabular}{|c|c|c|c|c|c|c|c|c|c|c|c|c|c|}
\hline \multirow{6}{*}{ 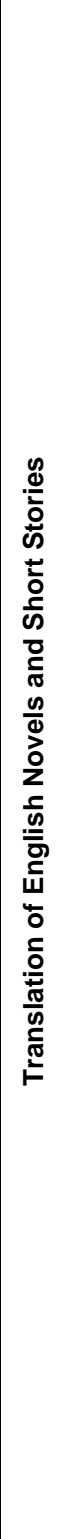 } & 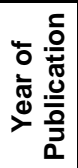 & $\begin{array}{l}\infty \\
\stackrel{\leftrightarrow}{\rightarrow}\end{array}$ & 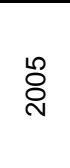 & $\begin{array}{l}\infty \\
\stackrel{\infty}{-} \\
\rightarrow\end{array}$ & 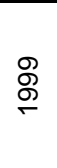 & ষ্ণ & ర్రి & $\stackrel{\text { ֻి }}{\text { ¿ }}$ & ¿̊̀ & 号 & 용 & 号 & $\vec{\sim}$ \\
\hline & 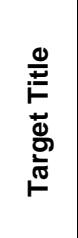 & $\stackrel{\widetilde{\pi}}{\widetilde{J}}$ & : & 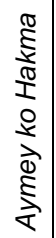 & 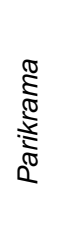 & $\frac{\frac{\pi}{d}}{\frac{\pi}{Q}}$ & 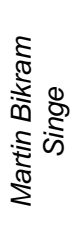 & 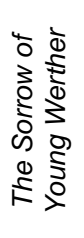 & 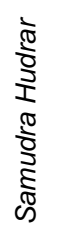 & 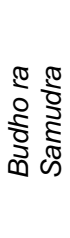 & 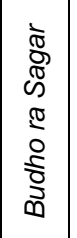 & 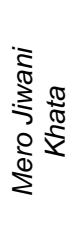 & 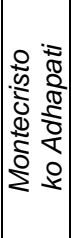 \\
\hline & $\begin{array}{l}\frac{\overline{0}}{0} \\
\frac{\pi}{0} \\
\frac{5}{\varpi} \\
\stackrel{5}{=}\end{array}$ & 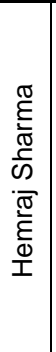 & 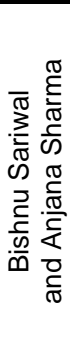 & 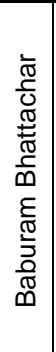 & 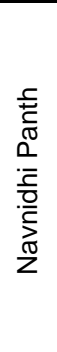 & 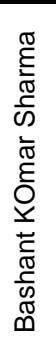 & 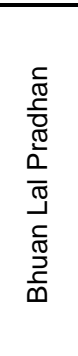 & 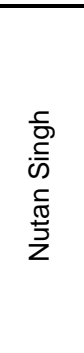 & 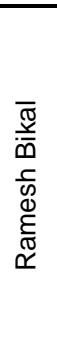 & 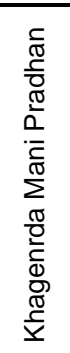 & 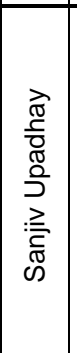 & 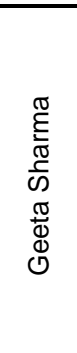 & 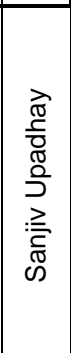 \\
\hline & 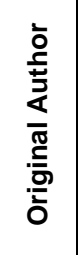 & 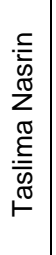 & 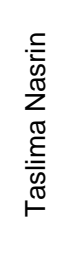 & 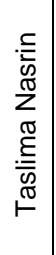 & 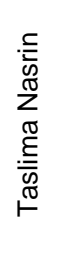 & 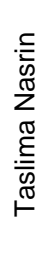 & 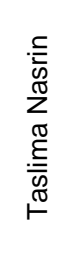 & $\begin{array}{l}\stackrel{0}{ \pm} \\
\stackrel{ \pm}{ \pm} \\
\stackrel{0}{0}\end{array}$ & 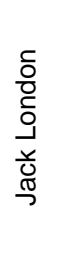 & 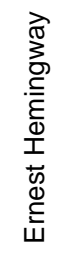 & 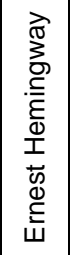 & 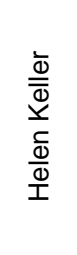 & 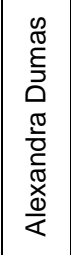 \\
\hline & 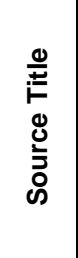 & $\stackrel{\widetilde{\pi}}{\widetilde{J}}$ & : & 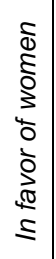 & 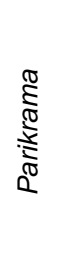 & $\frac{5}{\mathbb{Z}}$ & 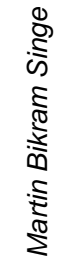 & 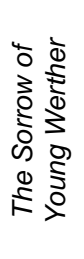 & 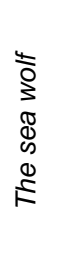 & 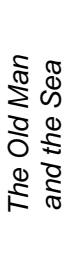 & 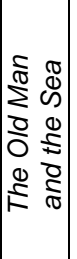 & 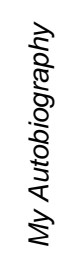 & 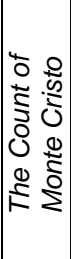 \\
\hline & $\begin{array}{l}\# \\
\bar{\omega}\end{array}$ & ட̊ & ถึ) & กิ & ณొ & 官 & 吕 & ட̊ & in & $\stackrel{\infty}{\llcorner}$ & ஜ & 8 & -10 \\
\hline
\end{tabular}


Most of the short story writers and novelists write in different languages like Russian, French, German, Bengali etc but the source language for the Nepali translations was English. To look at the lists of English translations, we can clearly observe the Russian writers like Anton Chekov, Maxim Gorky, Leo Tolstoy etc are translated in maximum number. The reason for translating their novels and short stories was the various themes like, Realism, Modernism, and Existentialism which captivated the minds of the Nepali translators. Apart from these Russian writers, most of the novels of Taslima Nasrin have been translated in Nepali to enrich the women writings in Nepali literature.

These writings would not have existed in Nepali without the help of the translators like Parasmani Pradhan, Krishna Prakash Shresta, Okima Gwyn etc. It was mainly their effort to introduce the best of the English writings to the Nepali readers and writers, as a result the Nepali literature experienced the new methods, genres and themes. Among the translators, Okima Gwyn is the writer of Nepali novel Sunakhari (Orchid, 1980) which was awarded by Sahitya Akadami in 1980.

\section{Translation of Dramas}

The drama in Nepali has been one of the neglected genres, but with the emergence of the dramatists like Lakshmi Prasad Devkota, Gopal Prasad Rimal, Balkrishna Sama, Madhab Bhandari, Basu Shashi and Man Bahadur Mukhiya, Nepali drama saw the best of the dramas and there were several tragedies, comedies and others related to the social issues. In terms of development, drama as a genre in Nepali literature owes a great deal to the translation which helped it to reach the pinnacle of success in the hands of the above dramatists. Most of these dramatists borrowed the methods from the 
translations of English dramas of Ibsen, Beckett, Brecht, Homer, Shakespeare etc. The contribution of Gopal Prasad Rimal and Balkrishna Sama towards the development of drama by using the methods of Ibsen and Shakespeare is noteworthy. Balkrishna Sama, who wrote 17 plays in Shakespearean style, breathed life into Nepali drama. He lifted Nepali drama to new heights in terms of its social background, philosophical depth, logicality and poetic dialogues. Let us now examine Balkrishna Sama's Mutuko Byatha, one of the first tragedies of Nepali literature, which is based on Shakespearean tragedy. The language of the drama was based on a common speech; the audience was able to relate the play with their personal life. He has adopted Marlow's blank verse in his tragedies.

Mutuko Vyatha is a social tragedy which deals with the autocratic rule of the Ranas in Nepal ${ }^{3}$. This tragedy takes the theme of unrequited love between separated lovers and sets in a recognizably Nepali idiom. Apart from being a first tragedy, it is written in a language which can be easily understood by the common people and in fact this was one of the purposes of his writings. The influence of Shakespearean play Macbeth (1623) can be seen in the following lines:

To-morrow, and to-morrow, and to-morrow, Creeps in this petty pace from day to day, To the last syllable of recorded time; And all our yesterdays have lighted fools The way to dusty death. Out, out, brief candle! Life's but a walking shadow, a poor player, That struts and frets his hour upon the stage, And then is heard no more. It is a tale Told by an idiot, full of sound and fury, Signifying nothing. (Shakespeare, 2007, Act V, Scene 5) 
Shakespeare uses blank verse in the above lines and the similar style is used by Sama in the following lines drawn from Mutuko Vyatha which is also written in the blank verse,

We rely upon tomorrow in this world,

Without realizing that we do so,

We consign yesterday to oblivion, and without pause

Today we say tomorrow: each and every tomorrow

Passes into yesterday. (Sama, 1903, Act iii, Scene 3)

The above lines show the resemblance in terms of matter and meter of Shakespearean play and the entire drama is modeled on the lines of the English tragedy. The entire drama is divided into 5 acts and 13 scenes which is typical of Renaissance dramas. The story of two lovers who suffer from the separation is very much related to Shakespeare's Romeo and Juliet. The influence and success of Mutuko Vyatha in Nepali theater led to the proliferation of tragedies in Nepali literature. The credit for this proliferation goes to the dramas that have been translated from English into Nepali which is given in the following pages: 


\begin{tabular}{|c|c|c|c|c|c|c|c|c|c|c|}
\hline \multirow{6}{*}{ 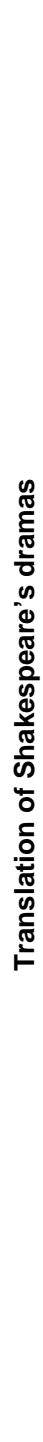 } & 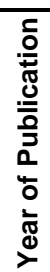 & $\begin{array}{l}\breve{~} \\
\stackrel{-}{7}\end{array}$ & 离 & 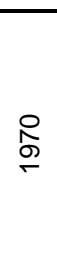 & 吕 & 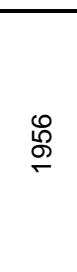 & \begin{tabular}{l}
0 \\
\multirow{9}{9}{}
\end{tabular} & 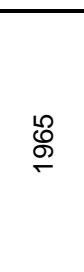 & న్ & $\begin{array}{l}0 \\
\stackrel{2}{9} \\
\end{array}$ \\
\hline & 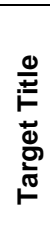 & $\begin{array}{l}\stackrel{\bigcirc}{\Phi} \\
\stackrel{\Xi}{\Xi}\end{array}$ & 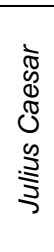 & 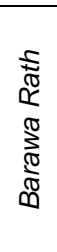 & 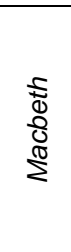 & 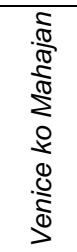 & 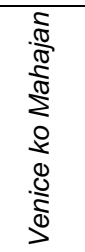 & 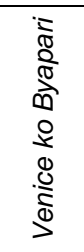 & 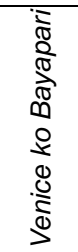 & $\begin{array}{l}\stackrel{\varpi}{\Xi} \\
\underset{\mathbb{\Xi}}{\mathbb{2}}\end{array}$ \\
\hline & 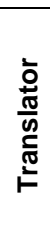 & 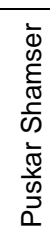 & 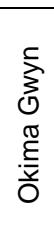 & 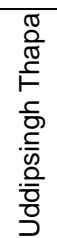 & 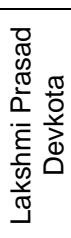 & 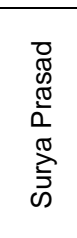 & 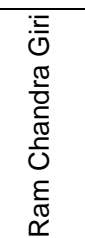 & 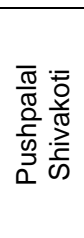 & 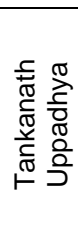 & 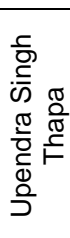 \\
\hline & 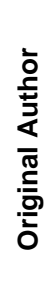 & 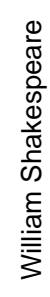 & 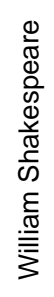 & 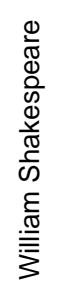 & 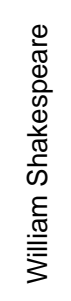 & 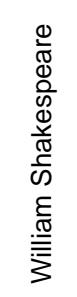 & 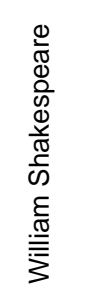 & 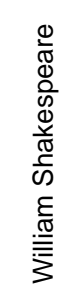 & 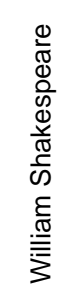 & 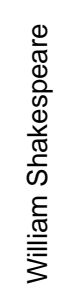 \\
\hline & 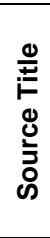 & $\begin{array}{l}\stackrel{Ð}{\Phi} \\
\stackrel{\Xi}{\Xi}\end{array}$ & 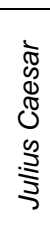 & 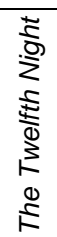 & $\begin{array}{l}\text { 志 } \\
\frac{\mathbb{U}}{\pi} \\
\sum\end{array}$ & 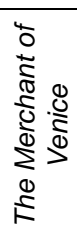 & 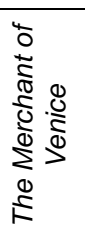 & 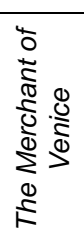 & 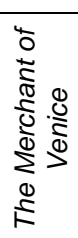 & 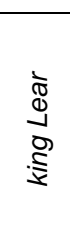 \\
\hline & $\begin{array}{l}\# \\
\dot{\omega}\end{array}$ & $r$ & $N$ & $m$ & $\nabla$ & เ & 0 & $r$ & $\infty$ & $\sigma$ \\
\hline
\end{tabular}


Shakespeare being the most influential dramatists, the present research has given a separate table for the translations of his plays alone. Apart from that, the tragedies of Shakespeare were adopted by the Nepali dramatists like Balkrishna Sama and Lakshman Sirmal. In fact, most of the dramas of Sama were modeled on the Shakespearean tragedy. Lakshman Sirmal's tragedy Cerfew (2007) received Sahitya Akademi Award in 2007 and he is the only dramatists to win this prestigious award. The Merchant of Venice was translated four times by different translator, to introduce the new form of drama that is tragic comedy. Because of these reasons and considering Shakespeare as the best dramatist of all the time, the present research has provided his dramas in the separate table.

However, the research does not deny the contribution of the other dramatists and their influence on Nepali literature. The table in the following page is the list of other English dramas which were translated from English into Nepali 


\begin{tabular}{|c|c|c|c|c|c|c|c|c|c|c|c|c|c|c|c|c|c|}
\hline & > & & 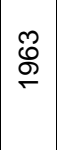 & 总 & 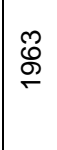 & 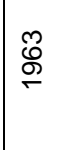 & 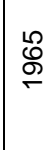 & $\begin{array}{l}\stackrel{8}{\circ} \\
\stackrel{2}{9} \\
\end{array}$ & $\begin{array}{l}\text { 怘 } \\
\stackrel{్}{\rightarrow}\end{array}$ & 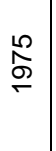 & $\begin{array}{l}\infty \\
\stackrel{\infty}{\sigma} \\
\stackrel{్}{\hookrightarrow}\end{array}$ & 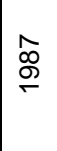 & $\begin{array}{l}\widehat{\infty} \\
\stackrel{\infty}{\rightarrow}\end{array}$ & 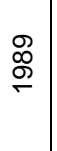 & $\begin{array}{l}\vec{\sigma} \\
\stackrel{ }{-}\end{array}$ & ने & 只 \\
\hline \multirow{5}{*}{ 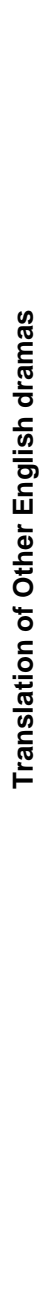 } & 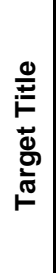 & $\begin{array}{l}0 \\
\frac{0}{0} \\
0 \\
\frac{0}{0} \\
\dot{0} \\
\text { in }\end{array}$ & 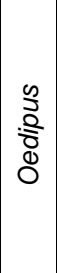 & $\underset{\frac{\pi}{U}}{\stackrel{\pi}{u}}$ & 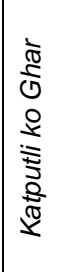 & 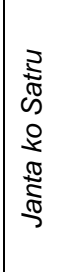 & 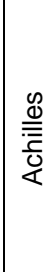 & 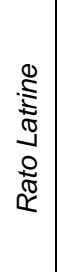 & 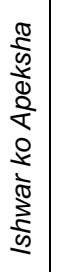 & 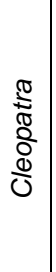 & 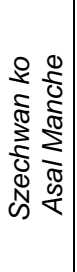 & 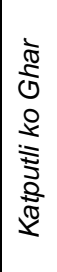 & 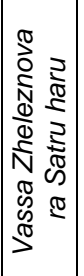 & 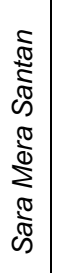 & 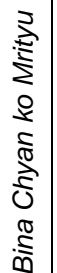 & 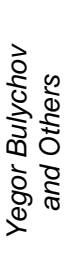 & 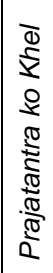 \\
\hline & 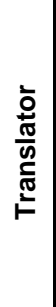 & 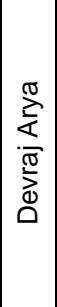 & 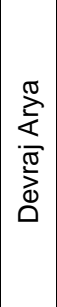 & 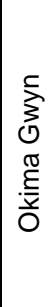 & 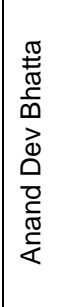 & 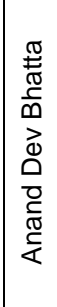 & 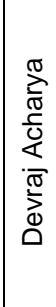 & 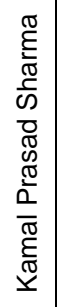 & 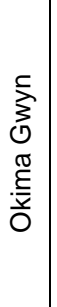 & 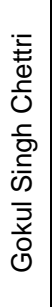 & 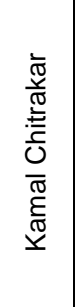 & 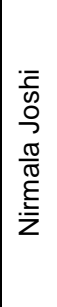 & 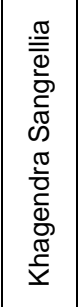 & 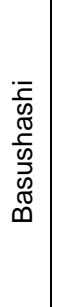 & 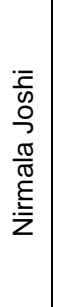 & 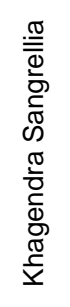 & 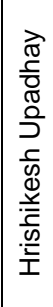 \\
\hline & 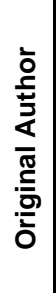 & $\begin{array}{l}0 \\
\frac{0}{0} \\
0 \\
\frac{0}{0} \\
\dot{0} \\
i\end{array}$ & $\begin{array}{l}0 \\
\frac{0}{0} \\
0 \\
\frac{0}{0} \\
\dot{0} \\
\text { is }\end{array}$ & 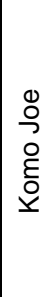 & 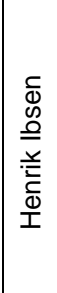 & 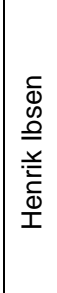 & 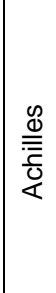 & 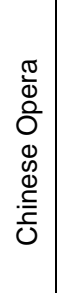 & 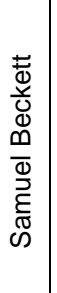 & 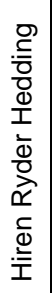 & 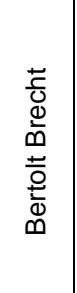 & 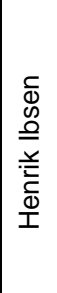 & 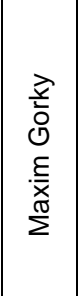 & 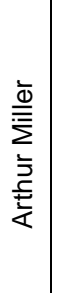 & 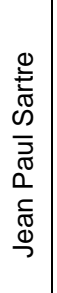 & 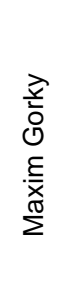 & 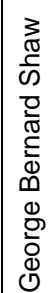 \\
\hline & 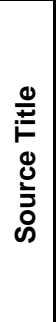 & 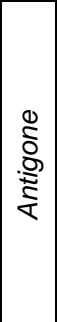 & 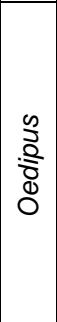 & $\overbrace{\frac{\pi}{U}}^{\pi}$ & 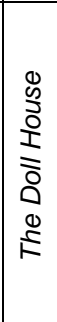 & 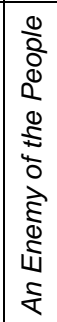 & $\begin{array}{l}\text { षे } \\
\text { है } \\
\text { ईे }\end{array}$ & $\begin{array}{l}\stackrel{0}{E} \\
\widetilde{\Xi} \\
\vec{\Xi} \\
\widetilde{\Xi} \\
\widetilde{\Xi}\end{array}$ & 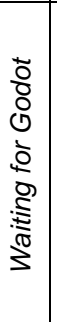 & 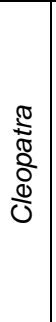 & 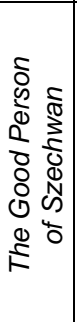 & 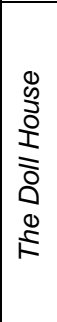 & 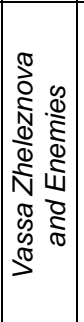 & 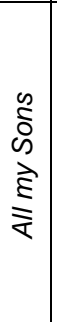 & 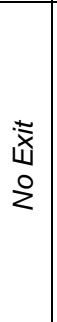 & 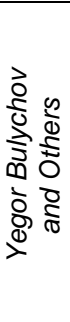 & 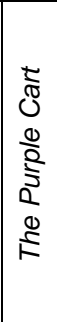 \\
\hline & $\neq$ & -1 & $N$ & $m$ & $\nabla$ & เ & 0 & $\sim$ & $\infty$ & $\sigma$ & 가에 & $\exists$ & Эิ & $\stackrel{m}{\rightarrow}$ & $\vec{\exists}$ & $\stackrel{\overbrace{}}{\neg}$ & $\stackrel{9}{-1}$ \\
\hline
\end{tabular}


Similar to the translations of English novels and short stories, the Russian dramas were translated the most in Nepali literature. The translation of Ibsen's The Doll House has been included in the curriculum of North Bengal University. The translation of Samuel Beckett's Waiting for Godot is held as the best translation in Nepali literature for translating such a complex theme like 'Theatre of Absurd'.

The idea of charting various translated genres in Nepali literature is to highlight the influential aspect of translation in building a national literature which is in the emerging phase. The major influences in Nepali have been the tragedies and comedies of William Shakespeare, which can be seen in the writings of Balkrishna Sama's Mutuko Byatha. The influence of modernist writers in shaping or giving a new format of writing prose poetry is another aspect which has enriched the entire school of Nepali poetry. The novel writing gave impetus to the writers to try with various western philosophical modes, especially Existentialism and Realism. Apart from philosophical aspect, the development of feministic writings is another major area of focus which is yet to be explored.

\section{Notes :}

1. Arnold's touchstone method is a comparative method of criticism. According to this method, in order to judge a poet's work properly, a critic should compare it to passages taken from works of great masters of poetry, and that these passages should be applied as touchstones to other poetry. Even a single line or selected quotation will serve the purpose. If the other work moves us in the same way as these lines and expressions do, then it is really a great work, otherwise not.

2. Leela lekhan, a phrase that stems from the Hindu understanding of leela as 'all of reality'. Leela Lekhan is based on the view that subjectivity dominates the human landscape from rationality to 
morality. While this can inevitably lead to a deceptive life, it does not negate the existence of an objective reality.

3. The period between 1846 to 1962 , is ruled by Ranas in Nepal who established a dictatorial rule. The worst phase in the history of Nepal.

\section{REFERENCES}

Baral, Krishna Hari and Netra Atom. 2001. Upanyas- Siddanta Ra Nepali Upanyas, Sajha Prakashan.

Basnet, Susan. 2007. Culture and Translation. In A Companion to Translation Studies. British Library Cataloguing in Publication Data, NY, 13-24.

Biswas, Sanjay and Samir Roka. 2005. Darjeeling Truth and Beyond. Darjeeling.

Catford, J.C. 2002. Translation Shifts. In The Translation Studies Reader. Routledge, New York, 141-148.

Chamberlain, Lori.1992. Gender and the Metaphorics of Translation. In Rethinking Translation Discourse, Subjectivity, Ideology. Routledge, London, 57-75.

Chesterman, Andrew. 2007. Bridge Concepts in Translation Sociology. In Constructing a Sociology of Translation. John Benjamins Publishing Company, Amsterdam, 171-187.

Dahal, Rajkumari. 2010. Anuvad Bimarsh. Pratima Prakshan,Siliguri.

Derrida, Jacques.1978. Structure, Sign, and Play in the Discourse of Human Science. In Writing and Difference. Trans. Routledge, Great Britain, 351371.

Desai, K Shantinath. 2007. Creative Aspect of Indian English. Sahitya Academi, New Delhi.

Elliot. T.S. 1934. The West Land and Other Poems. Harvest Book, USA.

Froster N Michael. 2008. Kant and Skepticism. Princeton University Press, Princeton.

Gayatri, Spivak. 2002. Politics of Translation. In The Translation Studies Reader. Routledge, New York, 397-417. 
History of English Translations and its Influence on Nepali Literature

Khagendra Mani Pradhan. 2010. The Old Man and the Sea. Trans. Sikkim Academy, Sikkim.

Gorkhapedia. Web. 13 July. 2012.

$<\underline{\text { http://thehimalayanbeacon.com/encyclopedia/tiki- }}$ index.php?page $=$ Indra + Bahadur+Rai $>$.

Gorkhapatra: The Rising Nepal. Web. 13 July. 2012. <http://www.gorkhapatra.org.np/gopa.detail.php?article_id=29647\&cat id $=14>$.

Hutt, Michael. 1997. Modern Literary Nepali: An Introductory Reader. Oxford University Press, Delhi.

Hutt, Michael. 1998. Nepali: A National Language and its Literature. Sterling Publishers, New Delhi.

Kant, Emmanuel. 1999. Critique of Pure Reason, Introduction. In Epistemology the Classical Reading. Blackwell, Oxford.

Karna Thami. 2011. Purkha. Trans. Sahitya Academy, New Delhi.

Mukherjee, Sujit. 1997. Translation as Discovery and Other Essays. Allied Publisher, New Delhi.

Munday, Jeremy. 2008. Introducing Translation Studies: Theories and Applications. Routledge, New York.

Nida, Eugine. 2002. "Principles of Correspondence.” Lawrence Venuti. The Translation Studies Reader. New York: Routledge, (126-141).

Parijat. 2010. Blue Mimosa. Trans. Orchid Books, Kathmandu.

Chitrabadhur Chettri. 1975. Boni. Trans. Nirman Prakshan, Sikkim.

Shresta, Dayaram. 2008. Sahitya ko Itihas : Sidhanta ra Sandhrva. Paluwa Prakshan, Kathmandu.

Simon, Sherry. 1996. Gender in Translation: Culture and Identity and the Politics of Transmission. Routledge, London.

Starr, Larry. 2002. The Dickenson Songs of Aaron Copland. The College Music Society, USA.

Subba, Bindya. 1999. Athah. Jaibharat Printing Press, Varanasi. 
Subba, Manprasad. 2010. Unfathomable. Trans. Sahitya Akademi, New Delhi.

Toury, Giddon. 2002. The Nature and Role of Norms in Translation. In The Translation Studies Reader. Routledge, New York, 79-92.

Von Flotow, Louise. 1997. Translation and Gender: Translating in the Era of Feminism. St. Jerome Publishing, Manchester. 